\title{
Mathematical vs. Experimental Stress Analysis of Inhomogeneities in Solids
}

\author{
W.H. Müller
}

Laboratorium für Technische Mechanik, Universität Paderborn (FB 10), Pohlweg 47-49, 33098 Paderborn, Germany

\begin{abstract}
The intent of this paper is to apply the discrete Fourier transform to the computation of eigenstress and eigenstrain fields around heterogeneities in composite materials. To this end the discrete Fourier transform is first briefly reviewed and then used to solve the basic equations of linear elasticity as pertinent to eigenstrained bodies under external loads. The results of this procedure are then used to discuss a few typical geometries such as an hexagonal two-dimensional array of thermally as well as elastically mismatched fibers in a composite matrix and a spherical Zirconia inclusion after a phase transformation. The resulting stress-strain fields are finally compared to experimental observations of eigenstress fields. The experimental techniques considered include photoelastic analyses as well as electron diffusion contrast techniques. It will be shown that the discrete Fourier transform as applied to eigenstress problems is capable of simulating the outcome of such experiments.
\end{abstract}

\section{INTRODUCTION}

The presence of inhomogeneities in solids very frequently generates eigenstresses and eigenstrains. A well known example are fiber-reinforced composite materials which generally show a thermal as well as an elastic mismatch between the fibres and the surrounding matrix material (e.g., Chermant and Despierres [1]). Other examples are ceramic materials reinforced by Zirconia particles which, during a martensitic phase transition, may transform considerably in shape and size (e.g., Stevens [2]) and, finally, superalloys (cf. e.g., Socrate and Parks [3]). In the latter case a so-called $\gamma$-phase develops in a $\gamma$-phase matrix. The lattice parameters of both phases are different and, consequently, high internal stresses and strains emerge in the vicinity of the coherent interface boundary. Moreover, in all examples it is possible to superimpose external mechanical stresses and strains on the solid. This may locally trigger the phase transformation and, over time, lead to morphological changes of the microstructure.

The experimental study and determination of eigenstresses and eigenstrains around precipitates has been subject of many investigations. Photoelasticity has been used by Ferber [4] who prepared two-dimensional macro-models in order to examine the stresses and strains around fiber cross-sections in cracked and uncracked fiber-reinforced materials. Electron diffraction contrast techniques were applied by Mader [5] to study in-situ the state of stress around Zirconia inclusions.

This paper attempts to mathematically model the experimentally observed stress-strain fields by using the discrete Fourier transform, as originally proposed by Khachaturyan [6] and Mura [7], or by Moulinec and Suquet [8]. This method has recently been applied successfully by Dreyer and Olschewski [9] to the micromechanical analysis of changes in morphology of textured solids. The method is capable of assessing the influence of anisotropy, thermal mismatch, ordering, lattice mismatch, particle interaction, as well as the resulting morphological development in a solid. In Section 2 some mathematical information on the discrete Fourier transform will be presented by juxtaposing it to the well-known integral Fourier transform. In Section 3 the discrete Fourier transform is used to determine the eigenstresses and eigenstrains 
in linear elastic heterogeneous bodies under external loads. Section 4 presents a brief discussion of the basic equations and results obtained in photoelasticity and electron diffraction when applied to eigenstressed materials. Finally, in Section 5 it will be shown how the discrete Fourier transform can be used to simulate such experiments on the computer.

\section{FOURIER TRANSFORMS - THEORY}

\subsection{Integral Fourier Transforms}

The center point of integral Fourier Transforms is Fourier's famous integral theorem which, for the purpose of this paper, can be summarized as follows (for a proof and a more detailed discussion see Morse and Feshbach [10] or Courant and Hilbert [11]):

Let $f$ be a function ${ }^{1}$ of position $x \in \mathbb{R}^{d}$, where $d=1,2,3$ for one- two-, or three-dimensional problems, such that:

$$
\int_{-\infty}^{+\infty}|f(x)| \mathrm{d} x<\infty
$$

Introducing the integral Fourier transform of $f$ :

$$
\hat{f}(\boldsymbol{k})=\frac{1}{(2 \pi)^{d / 2}} \int_{-\infty}^{+\infty} f(\boldsymbol{x}) \exp (\mathrm{i} \boldsymbol{k} \cdot \boldsymbol{x}) \mathrm{d} \boldsymbol{x} \equiv F[f(\boldsymbol{x})]
$$

it can be shown that it is equivalent to write:

$$
f(\boldsymbol{x})=\frac{1}{(2 \pi)^{d / 2}} \int_{-\infty}^{+\infty} \hat{f}(\boldsymbol{k}) \exp (-\mathrm{i} \boldsymbol{k} \cdot \boldsymbol{x}) \mathrm{d} \boldsymbol{k}, \text { where } f(\boldsymbol{x}) \text { is continuous }
$$

and:

$$
f(x) \rightarrow \frac{1}{2}(f(x+0)+f(x-0)), \text { where } f(x) \text { is discontinuous }
$$

The following useful rules for Fourier transforms should be mentioned, namely

(a) the differentiation theorem:

$$
F\left[\frac{\partial f}{\partial x_{j}}\right]=-\mathrm{i} k_{j} F[f(x)]
$$

(b) the integration theorem:

$$
\int_{-\infty}^{+\infty} f(\boldsymbol{x}) \overline{g(\boldsymbol{x})} \mathrm{d} \boldsymbol{x}=\int_{-\infty}^{+\infty} \hat{f}(\boldsymbol{k}) \overline{\hat{g}(\boldsymbol{k})} \mathrm{d} \boldsymbol{k}
$$

and

(c) the faltung theorem:

$$
F\left[\int_{-\infty}^{+\infty} f(x-y) g(y) \mathrm{d} y\right]=(2 \pi)^{d / 2} \hat{f}(\boldsymbol{k}) \hat{g}(\boldsymbol{k}) .
$$

\footnotetext{
${ }^{1}$ For example, the components of the strain or of the stress tensor.
} 


\subsection{Discrete Fourier Transforms ${ }^{2}$}

Consider a finite region $L^{d}$ in a space of dimension $d=1,2$, or 3 which is subdivided in $N^{d}$ equidistant cells of length $2 \pi / N$. Now define a discrete series $\left\{f_{\boldsymbol{\alpha}}\right\}$ for each of the discrete positions $\boldsymbol{x}(\boldsymbol{\alpha})=2 \pi / N \boldsymbol{\alpha}$ where $\boldsymbol{\alpha}=\left(\alpha_{1}, \ldots, \alpha_{d}\right)$ and $\alpha_{i}=\left(a_{i}+1, \ldots, a_{i}+N\right), i=1, \ldots, d$, such that $f_{\alpha+r N}=f_{\alpha}, \boldsymbol{r} \in \mathbb{Z}^{d}$ (periodicity condition). Finally define the discrete Fourier transform for this series:

$$
\hat{f}_{s}=\frac{1}{(N)^{d / 2}} \sum_{\alpha_{1}=1+a_{1}}^{N+a_{1}} \ldots \sum_{\alpha_{d}=1+a_{d}}^{N+a_{d}} f_{\alpha} \exp \left(\mathrm{i} \frac{2 \pi}{N} s \cdot \boldsymbol{\alpha}\right) \equiv Y\left[f_{\boldsymbol{\alpha}}\right], s_{i}=0,1, \ldots, N-1
$$

Then Fourier's theorem for a discrete periodic series can be written as follows:

$$
f_{\alpha}=\frac{1}{(N)^{d / 2}} \sum_{s_{1}=0}^{N-1} \ldots \sum_{s_{d}=0}^{N-1} \hat{f}_{s} \exp \left(-\mathrm{i} \frac{2 \pi}{N} s \cdot \alpha\right)
$$

As in the case of integral Fourier transforms a few useful rules can be established, such as

(a) the shifting theorem:

$$
Y\left[f_{\boldsymbol{\alpha}+\boldsymbol{\gamma}}\right]=\hat{f}_{s}=\exp \left(-\mathbf{i} \frac{2 \pi}{N} s \cdot \boldsymbol{\gamma}\right) Y\left[f_{\boldsymbol{\alpha}}\right]
$$

(b) the summation theorem:

$$
\sum_{\alpha_{1}=1+a_{1}}^{N+a_{1}} \ldots \sum_{\alpha_{d}=1+a_{d}}^{N+a_{d}} f_{\alpha} \bar{g}_{\alpha}=\sum_{s_{1}=0}^{N-1} \ldots \sum_{s_{d}=0}^{N-1} \hat{f}_{s} \overline{\hat{g}}_{s}
$$

and

(c) the faltung theorem:

$$
Y\left[\sum_{\gamma_{1}=1+a_{1}}^{N+a_{1}} \ldots \sum_{\gamma_{d}=1+a_{d}}^{N+a_{d}} f_{\alpha-\gamma} g_{\gamma}\right]=(N)^{d / 2} \hat{f}_{s} \hat{g}_{s}
$$

The shifting theorem can be used to compute the discrete Fourier transform of differential quotients of a function $f$ whose values coincide with those of the series $\left\{f_{\alpha}\right\}$ in the points $\boldsymbol{x}(\boldsymbol{\alpha})$ :

$$
\begin{aligned}
& Y\left[\frac{\partial f}{\partial x_{r}}\right]=\frac{1}{\mathrm{i} h} \sin \left(h s_{r}\right) Y[f]+\mathrm{O}\left(h^{2}\right) \equiv \xi_{r} \hat{f}+\mathrm{O}\left(h^{2}\right), h=\frac{2 \pi}{N} \\
& Y\left[\frac{\partial^{2} f}{\partial x_{r} \partial x_{r}}\right]=\frac{2}{h^{2}}\left(\cos \left(h s_{r}\right)-1\right) Y[f]+\mathrm{O}\left(h^{2}\right) \equiv \xi_{r r} \hat{f}+\mathrm{O}\left(h^{2}\right) \\
& Y\left[\frac{\partial^{2} f}{\partial x_{r} \partial x_{s}}\right]=\frac{1}{2 h^{2}}\left(\cos \left(h\left(s_{r}+s_{s}\right)\right)-\cos \left(h\left(s_{r}-s_{s}\right)\right)\right) Y[f]+\mathrm{O}\left(h^{2}\right) \equiv \xi_{r s} \hat{f}+\mathrm{O}\left(h^{2}\right), r \neq s .
\end{aligned}
$$

\footnotetext{
${ }^{2}$ In this section we follow the nomenclature used by Dreyer and Olschewski [8].
} 


\section{FOURIER TRANSFORMS - APPLICATION TO EIGENSTRAIN PROBLEMS}

\subsection{Formulation of the problem ${ }^{3}$}

The use of integral Fourier transform as well as Fourier series in elasticity problems has been demonstrated successfully by Mura [7]. Additional applications of the discrete Fourier transform can be found in the book by Khacharturyan [6] and, more recently, in the work by Moulinc and Suquet [8] or Dreyer and Olschewski [9]. Consider a heterogeneous body under the influence of external forces, $f_{i}^{0}$, $i=1, \ldots, d$. The heterogeneities are characterized by a stiffness matrix, $C_{i j k l}(x)$, and by a misfit, $\varepsilon_{i j}^{*}(x)$, where both sets of fields are functions of position, $x$. The stresses, $\sigma_{i j}$, and the total strains, $\varepsilon_{i j}$, are linked to each other by Hooke's law:

$$
\sigma_{i j}=C_{i j k l}\left(\varepsilon_{k l}-\varepsilon_{k l}^{*}\right)
$$

where the total strain can be expressed by the spatial derivative of the displacement vector, $u_{i}$, as follows:

$$
\varepsilon_{i j}=\frac{1}{2}\left(\frac{\partial u_{i}}{\partial x_{j}}+\frac{\partial u_{j}}{\partial x_{i}}\right)
$$

If, in particular, cubic symmetry of the body is assumed the following relation holds:

$$
C_{i j k l}=\lambda \delta_{i j} \delta_{k l}+\mu\left(\delta_{i k} \delta_{j l}+\delta_{i l} \delta_{j k}\right)+\mu^{\prime} \delta_{i j k l}
$$

where $\delta_{i j}$ denotes the Kronecker symbol and $\delta_{i j k l}$ is equal to one if all of its components are the same and zero otherwise. The symbols $\lambda, \mu$, and $\mu^{\prime}$ refer to the three elastic constants of cubic materials.

Moreover, the body is assumed to be in mechanical equilibrium, i.e., for its interior:

$$
\frac{\partial \sigma_{i j}}{\partial x_{j}}=0
$$

and on its surface:

$$
\sigma_{i j} N_{j}=f_{i}^{0}
$$

In order to determine the stresses the analysis will be carried out in two steps: First, the elastically homogeneous case will be considered, i.e., $C_{i j k t}$ is a constant. The elastically inhomogeneous case will be treated in a second step by means of the equivalence method developed by Mura and Eshelby [7].

\subsection{The elastically homogeneous body}

The stresses in a homogeneous body under external loads and eigenstrains can be determined by superposition of the following two cases, representing external loading by tension or compression, $f_{i}^{0}$, and pure eigenstrains, $\varepsilon_{i j}^{T}$, respectively:

$$
\varepsilon_{i j}^{0}: f_{i}^{0} \neq 0, \varepsilon_{i j}^{*}=0 \text { and } \varepsilon_{i j}^{T}: f_{i}^{0}=0, \varepsilon_{i j}^{*} \neq 0
$$

For example, in the case of plane strain the following result is obtained for the first problem by solving eqns (3.1.4-5) in connection with eqns $(3.1 .1,3)$ :

\footnotetext{
${ }^{3}$ In this section we follow the nomenclature employed by Dreyer and Olschewski [8].
} 


$$
\varepsilon_{11}^{0}=\frac{\left(\lambda+2 \mu+\mu^{\prime}\right) f_{1}^{0}-\lambda f_{2}^{0}}{\left(\lambda+2 \mu+\mu^{\prime}\right)^{2}-\lambda^{2}}, \varepsilon_{22}^{0}=\frac{\left(\lambda+2 \mu+\mu^{\prime}\right) f_{2}^{0}-\lambda f_{1}^{0}}{\left(\lambda+2 \mu+\mu^{\prime}\right)^{2}-\lambda^{2}}
$$

from which the resulting stresses can easily be computed using eqn (3.1.1).

In order to solve the eigenstrain problem the discrete Fourier transform of eqn (2.2.1) is applied to eqns $(3.1 .1,2,4)$ in connection with eqns $(2.2 .6-8)$ to yield:

$$
\hat{\sigma}_{i j}=C_{i j k l}\left(\hat{\varepsilon}_{k l}^{T}-\hat{\varepsilon}_{k l}^{*}\right), \quad \hat{\varepsilon}_{i j}^{T}=\frac{1}{2}\left(\xi_{j} \hat{u}_{i}+\xi_{i} \hat{u}_{j}\right), C_{i j k l} \xi_{j l} \hat{u}_{k}=C_{i j k l} \xi_{j} \hat{\varepsilon}_{k l}^{*}
$$

Introducing:

$$
M_{i k} \equiv C_{i j k l} \xi_{j l}, R_{i} \equiv C_{i j k l} \xi_{j} \hat{\varepsilon}_{k l}^{*}
$$

the last equation can be solved as follows:

$$
\hat{u}_{i}=M_{i r}^{-1} R_{r} \equiv \frac{1}{D} N_{i r} R_{r}=\frac{1}{D} N_{i r} C_{r s k l} \xi_{s} \hat{\varepsilon}_{k l}^{*} \equiv G_{i k l} \hat{\varepsilon}_{k l}^{*}, D \equiv \operatorname{det} M
$$

The new symbols used in this equation can be computed by straightforward matrix inversion after the stiffness matrix has been specified. For example, for a cubic material (see eqn (3.1.3)) the first component of $N_{i r}$ reads for the three-dimensional case:

$$
N_{11}=\left(a_{2} \xi_{22}+\mu \operatorname{tr} \xi\right)\left(a_{2} \xi_{33}+\mu \operatorname{tr} \xi\right)-a_{3}^{2} \xi_{23}^{2}, a_{2}=\lambda+\mu+\mu^{\prime}, a_{3}=\lambda+\mu
$$

Consequently, the eigenstrains, $\varepsilon_{i j}^{T}$, of eqn (3.2.1) can be computed as follows:

$$
\hat{\varepsilon}_{i j}^{T}=\hat{A}_{i j k l} \hat{\varepsilon}_{k l}^{*}, \hat{A}_{i j k l} \equiv \frac{1}{2}\left(\xi_{j} G_{i k l}+\xi_{i} G_{j k l}\right)
$$

\subsection{The elastically inhomogeneous body}

Following the equivalent inclusion method as outlined in Mura [7], Chapter 4, the total strain, $\varepsilon_{i j}$, in a heterogeneous body with a spatially varying stiffness matrix under external load can be approximated using the following iteration scheme (also see Dreyer and Olschewski [9]):

$$
\begin{aligned}
& \varepsilon_{i j}=\varepsilon_{i j}^{0}+\varepsilon_{i j}^{1}+\varepsilon_{i j}^{2}, \quad \varepsilon_{i j}^{1}=Y^{-1}\left[\hat{A}_{i j r s} \hat{\varepsilon}_{r s}^{*}\right], \quad \varepsilon_{i j}^{2}=Y^{-1}\left[\hat{A}_{i j r s} \hat{\varepsilon}_{r s}^{H}\right] \\
& \stackrel{(n+1)}{\varepsilon}{ }_{i j}^{H}=-\left(C^{-1}\right)_{i j m n}^{+}\left(C_{m n o p}^{-}-C_{m n o p}^{+}\right) S\left(\varepsilon_{o p}^{0}+Y^{-1}\left[\hat{A}_{o p r s} \hat{\varepsilon}_{r s}^{*}\right]-\varepsilon_{o p}^{*}\right) \\
& -\left(C^{-1}\right)_{i j m n}^{+}\left(C_{m n o p}^{-}-C_{m n o p}^{+}\right) S Y^{-1}\left[\hat{A}_{o p r s} \stackrel{(n)}{\hat{\varepsilon}_{r s}^{H}}{ }_{r s}\right]
\end{aligned}
$$

where "+" and "-" refer to properties of the matrix and the heterogeneity, respectively, and $S$ denotes an order parameter with the following property:

$$
S=\left\{\begin{array}{ll}
1, & \text { if } x \in \mathbb{R}^{-} \\
0, & \text { if } x \in \mathbb{R}^{+}
\end{array},\right.
$$

and:

$$
\stackrel{(0)}{\varepsilon} \underset{r s}{H}=0
$$




\section{EXPERIMENTAL ANALYSIS OF EIGENSTRAIN PROBLEMS}

\subsection{Photoelasticity}

Photoelasticity has been used by Ferber [4] to visualize the eigenstresses and eigenstrains around fibers in thermally mismatched fiber-reinforced materials. To this end, two-dimensional models were made and analyzed in a polariscope. Figure 1 shows typical isochromates in a circular matrix (diameter $=150 \mathrm{~mm}$ ) made of Araldite $\mathrm{F}$ (Young's modulus $E=2583 \mathrm{MPa}$, Poisson's ratio $\mathrm{v}=0.4$, thermal expansion coefficient $\left.\alpha=37.5 \cdot 10^{-6} 1 / \mathrm{K}\right)$ which contains six pellets made of steel $(E=210000 \mathrm{MPa}, v=0.3$, $\left.\alpha=12 \cdot 10^{-6} 1 / \mathrm{K}\right)$. The specimen was subjected to cooling in a temperature chamber from $+60^{\circ} \mathrm{C}$ down to $-7.5^{\circ} \mathrm{C}$. Figure 2 shows the experimentally observed stresses along the $x$-axis of the specimen (see inset).

The normalized intensity $I_{H} / I_{A}$ of the isochromates can be computed from (cf., Ferber [4], pp. 40-41):

$$
\frac{I_{H}}{I_{A}}=\sin ^{2}\left(\frac{B}{S}\left\{\sigma_{12}^{2}+\frac{\left(\sigma_{11}-\sigma_{22}\right)}{4}\right\}^{1 / 2}\right), \frac{B}{S}=\frac{5}{18 \mathrm{MPa}} .
$$

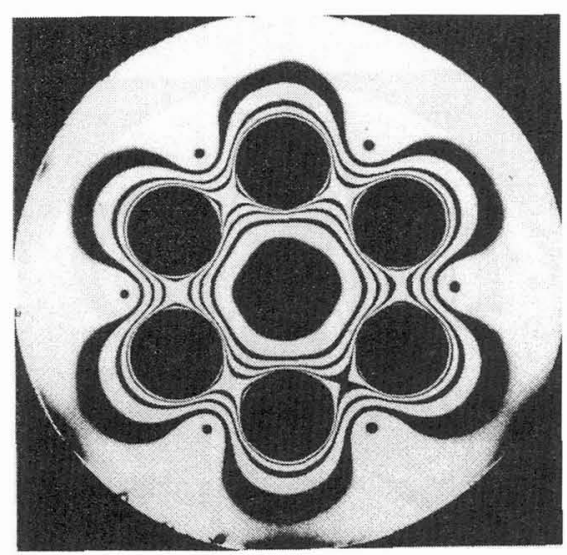

Figure 1: Isochromates of a composite model, Ferber [4]

\subsection{Electron diffraction contrast}

Figure 2: Distribution of stresses in the specimen of Fig. 1, [4]

Mader [5] used dynamic dark field electron transmission microscopy to visualize the in-situ stress-strain behavior around thermally mismatched tetragonal Zirconia inclusions of nearly spherical shape in an Alumina matrix. Figure 3 shows a typical result from his work. It should be pointed out that the characteristic contrast fringes around the inclusions do not directly represent the stresses nor the strains in the vicinity of the mismatched inclusion. Rather they are linked to a part of the strain tensor, e.g., $u_{x, z}$, in a somewhat "integral" manner. For example, in the case of a centrally located inclusion in a thick specimen the intensity of the contrast fringes can be computed from: 


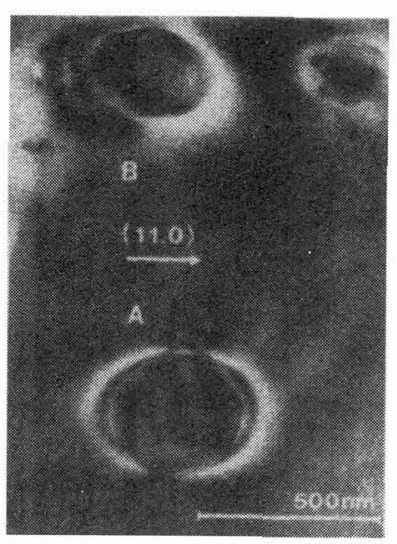

$$
I_{n}=\frac{1}{2}\left( \pm \cos \left(2 \pi P_{r}\right)+\cosh \left(2 \pi P_{i}\right)\right)
$$

and:

$$
P_{r}=\sigma_{r} \int\left(1+\left(\xi_{g}|g| u_{x, z}\right)^{2}\right)^{1 / 2} \mathrm{~d} t, P_{i}=\sigma_{i} \int\left(1+\left(\xi_{g}|g| u_{x, z}\right)^{2}\right)^{-1 / 2} \mathrm{~d} t .(4.2 .2)
$$

where $t$ denotes the thickness of the specimen, $\sigma_{r / i}$ are the so-called extinction numbers, $\xi_{g}$ is the extinction distance, and $|g|$ is the length of the diffraction vector.

Here it may suffice to say that by means of the electron diffraction contrast method the three-dimensional aspects of the eigenstress fields around heterogeneities are emphasized.

Figure 3: Dynamic dark-field TEM, Mader [5]

\section{RESULTS}

\subsection{Two-dimensional simulations}

Figure 4 presents the numerical simulation for the isochromates of the specimen shown in Figure 1. The results are based on the discrete Fourier transform when applied to a grid of size $256 \times 256$ pixels. The elastic constants of the inclusions and of the matrix were assumed to be equal and the misfit to be isotropic: $\varepsilon_{i j}^{*}=0.00249 \delta_{i j}$.

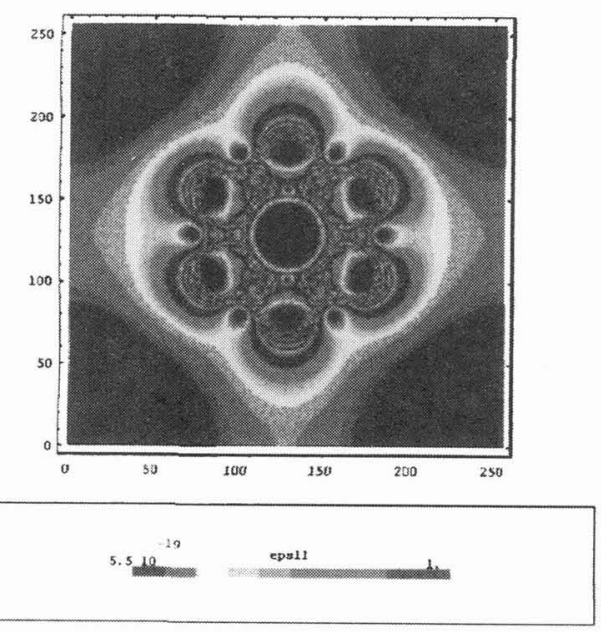

Figure 4: Simulation of isochromates

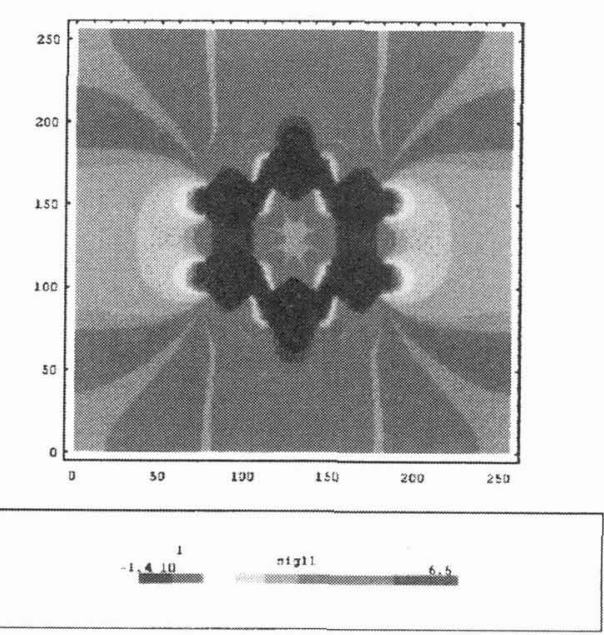

Figure 5: Stress distribution $\sigma_{11}$

Figure 5 presents the spatial distribution of the stress component, $\sigma_{11}$, in form of a density plot and Figure 6 shows the distribution of stresses, $\sigma_{11}$ and $\sigma_{22}$, along the horizontal line $x$ shown in Figure 2 (in units of MPa). The qualitative resemblance to the experimental results is remarkable. However, there is a major quantitative discrepancy between the experimental and numerical data. This is mainly due to the fact that the difference in elastic constants between the Araldite matrix and the steel pellets has been ignored completely. 

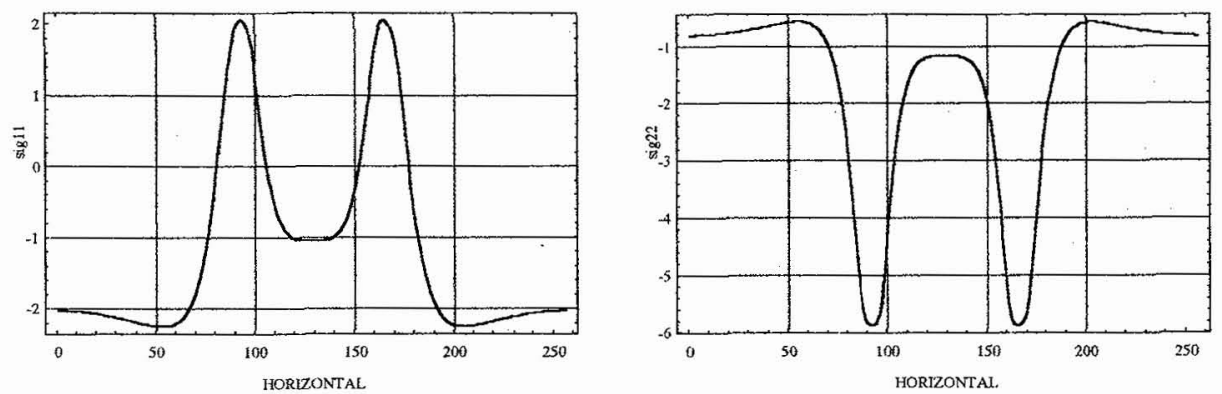

Figure 6: Distribution of stresses along the horizontal line $x$ of Figure 2.

It should be noted that the difference of the elastic constants of Araldite and steel is extremely large (see the data in the previous section). Therefore it is not sufficient to apply eqn (3.3.1) which is only a first order approximation for the total strain with regards to the difference in elastic mismatch. As an alternative one may turn to higher order approximations which, in fact, follow from the solution of an integral equation for the strains by means of the Neumann iteration scheme (cf., Mura [7] or Dreyer and

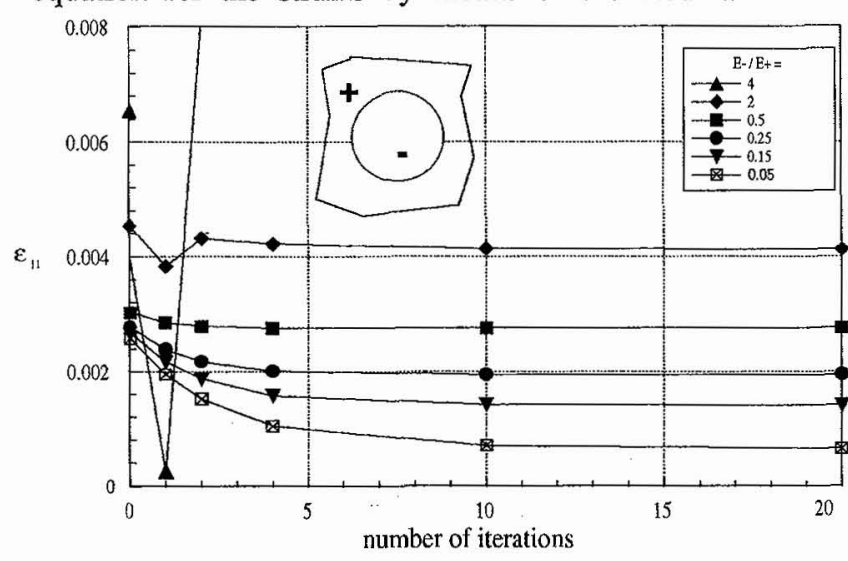
Olschewski [9]). Such higher order approximations are analyzed in Figure 7 which shows the maximum of the numerically predicted strain component, $\varepsilon_{11}$, for a single (= small) elastically and thermally mismatched cylinder. These values can be compared with an analytical solution (see, e.g., Müller and Schmauder [12]). It turns out that the Neumann iteration leads to convergence provided the elastic mismatch is not too large, as it is unfortunately the case for the specimen shown in Figure 2. To improve the convergence behavior of the presented method is left to future research.

Figure 7: Convergence of strain for various stiffness ratios
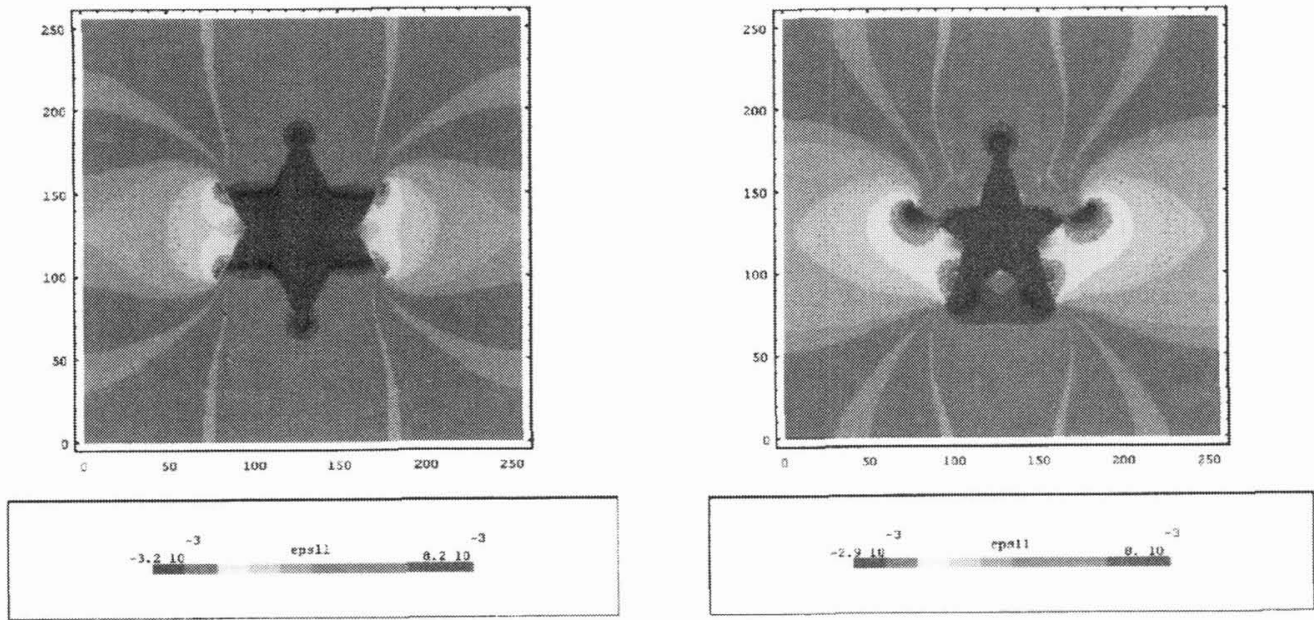

Figure 8: Discrete Fourier solution of the "Star of David" and the "Pentagonal Star" problem (Mura et al. [13]) 
The use of the discrete Fourier technique in the case of inclusions of complicated shape is demonstrated in the sequence shown in Figure 8 which presents the "Star of David" and the "Pentagonal Star" problems recently analyzed by Mura et al. [13]. The uniformity of the elastic field, $\varepsilon_{11}$, in the interior of a single (= small) star is clearly visible.

\subsection{Three-dimensional simulations}

As a first test toward the simulation of TEM experiments, such as the one shown in Figure 3, the strain fields $\varepsilon_{11}$ and $\varepsilon_{13}$ in the equatorial plane of a spherical isotropic inclusion were computed numerically. In this case an analytical solution is known (Mura [7]) and can be used for comparison. The grid used for discretization consisted of $128 \times 128 \times 128$ pixels and the numerical result is shown in Figure 9 . It is in good agreement with the analytical formula. Furthermore note that the viewgraph for $\varepsilon_{13}$ bears already a certain ressemblance to the dark field image shown in Figure 3. This is not surprising since for a sphere in an infinite body the following relation holds:

$$
\varepsilon_{13}=u_{1,3}
$$

the latter being the quantity responsible for the electron diffraction contrast (cf., eqn (4.2.2)).
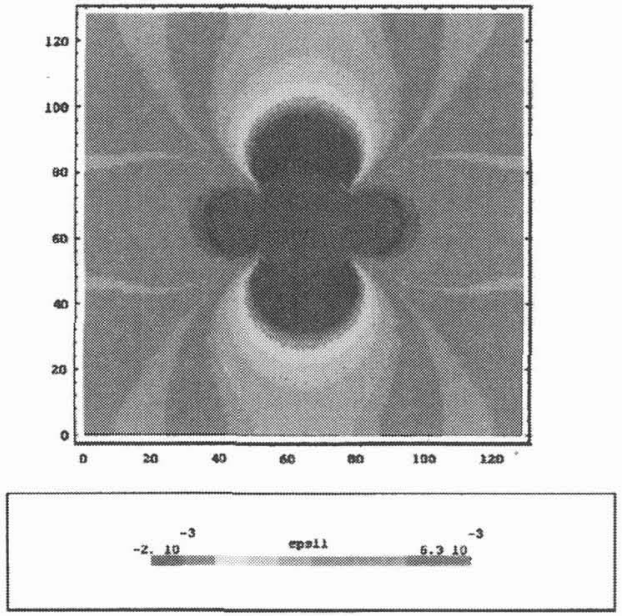
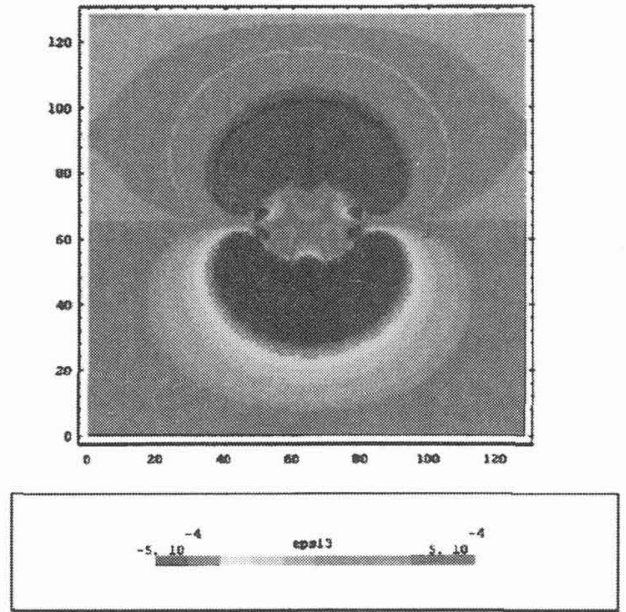

Figure 9: Discrete Fourier image for a spherical Zirconia inclusion

\section{CONCLUSIONS AND OUTLOOK}

It was shown how discrete Fourier transforms can be used to solve eigenstress problems in heterogeneous linear elastic materials. Numerical results were compared to experimental stress and strain data and, in particular, it was shown how discrete Fourier transforms can be used to model photoelastic and TEM experiments. So far the application of discrete Fourier transforms allows to compute the stress/strain behavior of moderately elastically mismatched materials. Further research is underway to cover the case of strong elastic mismatch. 


\section{Acknowledgment}

The author wishes to thank the Kommission für Forschung und wissenschaftlichen Nachwuchs der Universität Paderborn for financial support and Dr. W. Dreyer and J. Olschewski (BAM Berlin) for their advice and many helpful discussions.

\section{References}

[1] Chermant, J.-L., Despierres, T., "Ceramic Matrix Composites: Mechanical Behavior," Int. Symp. „Brittle Matrix Composites 4,” Warsaw, Sept. 13-15, 1994, editors: A.M. Brandt, V.C. Li and I.H. Marshall (IKE and Woodhead Publ., Warsaw, 1994) pp. 579-592.

[2] Stevens, R., An Introduction to Zirconia - Zirconia and Zirconia Ceramics (Magnesium Elektron Ltd, second Edition, Magnesium Elektron Publication No. 113, Litho 2000, Twickenham, UK, 1986).

[3] Socrate, S., Parks, D.M., Acta. metall. mater., 41 (7) (1994) 2185-2209.

[4] Ferber F., Bruchmechanische Analyse der Entstehung und Ausbreitung von Matrix- und Grenzflächenrissen in thermisch belasteten Faserverbundwerkstoffmodellen (Dissertation, Universität Paderborn, 1986).

[5] Mader, W., Phil. Mag. A, 55 (1) (1987) 59-83.

[6] Khacharturyan, A.G., Theory of Structural Transformations in Solids (John Wiley \& Sons, New York, Chichester, Brisbane, Toronto, Singapore, 1983).

[7] Mura, T., Micromechanics of Defects in Solids (Martinus Nijhoff Publishers, Dordrecht, second revised edition, 1987).

[8] Moulinec, H., Suquet, P., C. R. Acad. Sci. Paris, t. 318, Série II, pp. 1417-1423, 1994.

[9] Dreyer, W., Olschewski, J., personal communication.

[10] Morse, P.M., Feshbach, H., Methods of theoretical physics, part I (McGraw-Hill Book Company, New York, Toronto, London, 1953) pp. 453-467.

[11] Courant, R., Hilbert, D., Methoden der Mathematischen Physik I (Springer Verlag Berlin, Heidelberg, New York, 1968) pp. 65-70.

[12] Müller, W.H., Schmauder, S., Comp. Struct., 24 (1991) 1-21.

[13] Mura, T., Shodja, H.M., Lin, T.Y., "The Determination of the Elastic Field of a Pentagonal Star Shaped Inclusion," Twelfth U.S. National Congress of Applied Mechanics, June 27-July 1, 1994, Abstracts of Contributed Papers (University of Washington, Seattle, USA) p. 258. 